\title{
Soap-thickener induced local pressure fluctuations in a grease-lubricated elastohydrodynamic point contact
}

\author{
H Åström \\ Department of Mechanical Engineering, Linköping University, Sweden \\ C H Venner \\ Faculty of Mechanical Engineering, University of Twente, Enschede, The Netherlands
}

In several experimental investigations of grease-lubricated elastohydrodynamic (EHD) contacts indications of soap-thickener formations that enter the contact area have been reported, for example by Kageyama et al. (1), Cann and co-workers (2-4) and Aström and co-workers $(\mathbf{5}, 6)$. While passing through a contact such soap-thickener lumps significantly disturb the film thickness by locally increasing the surface deformation. These film-thickness fuctuations must be accompanied by pressure fluctuations, knowledge of which is essential to increase insight in the phenomena determining service life and emitted noise of grease-lubricated contacts. In this paper the authors present a combined experimental/numerical approach to generate insight into such pressure fluctuations. From a fringe pattern obtained with optical interferometry (ball-on-disc apparatus) a film-thickness map is created employing image analysis. This map serves as input to a numerical algorithm for the calculation of the pressure from force balance and elastic deformation theory. Consequently, no assumptions about the rheological behaviour of grease can be made.

The method was first tested out on an oil-lubricated contact. This test gave essential insight into the accuracy of the method proposed here and in the magnitude of surface texture induced pressure fluctuations. Subsequently the approach was successfully used to estimate the pressure variations resulting from soap-thickener formations in a grease-lubricated contact (between the same ball and disc).

\section{NOTATION}

a radius Hertzian contact circle $=\left\{\left(3 F R_{x}\right) /\left(2 E^{\prime}\right)\right\}^{1 / 3}$

$d \quad$ intergrid spacing

E elasticity modulus

$E^{\prime} \quad$ reduced modulus of elasticity, $2 / E^{\prime}=\left(1-v_{1}^{2}\right) / E_{1}$ $+\left(1-v_{2}^{2}\right) / E_{2}$

$F \quad$ load

$G$ parabolic approximation of the surface separation $=X^{2} / 2+Y^{2} / 2$

$h \quad$ film thickness

$H$ dimensionless film thickness $=h R_{x} / a^{2}$

$H_{00}$ integration constant

$n$ refractive index

$p$ pressure

$p_{\mathrm{h}} \quad$ maximum Hertzian pressure $=(3 F) /\left(2 \pi a^{2}\right)$

$\boldsymbol{P}$ dimensionless pressure $=p / p_{\mathrm{h}}$

$q \quad$ fringe order

$\boldsymbol{R}_{\boldsymbol{x}} \quad$ reduced radius of curvature

$x \quad$ coordinate in direction of flow

$X$ dimensionless coordinate $=x / a$

$y$ coordinate

$Y$ dimensionless coordinate $=y / a$

$\lambda \quad$ wavelength

$v \quad$ Poisson's ratio

$\Delta \phi \quad$ phase shift difference

\section{INTRODUCTION}

Elastohydrodynamic (EHD) lubrication is the type of lubrication prevailing in so-called concentrated contacts, for example the contact between two tooth flanks in a toothed transmission gear or between rolling element and raceway in a rolling element bearing. As lubricants, mineral oil and lubricating greases are most

The MS was received on 29 October 1993 and was accepted for publication on 20 May 1994.

J02493 C IMechE 1994 widely used. This choice naturally depends on the specifics of the contact, for example the environment in which it operates. For example, most rolling element bearings are lubricated with grease. In comparison with oil, grease has the advantage of staying at the lubricating place, thereby acting as a seal, that is protecting the contact against external contaminations. In addition, it more efficiently damps vibrations that might occur in the bearing. Disadvantages, on the other hand, are a low cooling capacity and, partly as a result of that, a limited use for high-speed applications.

Greases are semi-liquid lubricants consisting of a lubricating oil, a thickener of some kind and additives. The most commonly used thickener at present is soap thickener which creates a structure of solid fibres in the oil. This network of fibres retains the oil by capillary forces and other physical bonds and gives the grease its consistency. Depending on the composition of the grease and on the manufacturing process, the soapthickener fibres are gathered in larger or smaller formations in the grease rather than being equally distributed. Hence, greases are not homogeneous on a scale comparable to the lubricating film thickness found in rolling element bearings.

Reviewing the tribological literature, it shows that, over the last few decades, EHD lubrication has received much attention, both from a theoretical and from an experimental point of view. The theoretical studies usually involve the numerical solution of pressure and film thickness in the contact, using some physical mathematical model built around the Reynolds equation to describe the flow and the theory of elastic deformation of semi-infinite bodies. The resulting algorithms were initially used to perform parameter studies, that is investigating the dependence of film thickness or traction on lubricating properties and load conditions, assuming smooth surfaces.

The last decade has seen great improvement in 
numerical algorithms and as a result increasingly complex simulations are performed now, for example investigation of surface features and related transient effects. On the other hand, the physical mathematical models employed in the studies have changed relatively little. Generally the lubricant is assumed to be fully liquid, for example oil, and it is characterized by a viscosity and a density, both depending on pressure and/or temperature. More complex lubricant behaviour has been considered but only by introducing quantities like an effective viscosity and density, to ensure that Reynolds equation in one form or the other can still be used. Examples of this are numerical calculations on grease-lubricated EHD contacts; see, for example, Dalmaz and Chaomleffel (7), Bordenet et al. (8), Dong and Qian (9) and Jonkisz and Krzeminski-Freda (10, 11), where the Reynolds equation is used and the grease is regarded as a fluid.

However, from the above description of grease behaviour it seems quite unlikely that their rheological behaviour can be accurately modelled in this way, let alone that the behaviour of a grease EHD lubricated contact can be predicted accurately. No doubt this has contributed to the fact that most investigation dealing with grease-lubricated EHD contacts are experimental. In fact, the many experimental results that have been published do confirm the complex nature of grease behaviour and grease lubrication mentioned above. For example, in some cases evidence of formations of thickeners entering the concentrated contact have been found. Kageyama et al. (1) observed local fluctuations in the film thickness of their grease-lubricated point contact. Cann and co-workers (2-4) detected a layer of soap fibres in the track behind their grease-lubricated point contact, indicating that soap-fibre formations enter and pass the contact area. Åström and co-workers $(5,6)$ observed local film thickness fluctuations in their point contact lubricated with grease. The fluctuations were caused by soap-thickener formations that entered and passed the EHD contact.

One of the techniques used in these experiments is optical interferometry measurement of the film thickness in the contact. A typical set-up is the ball and disc machine, where a steel ball runs against a glass or sapphire disc. The earliest machines of this type date back to the 1960s; for example see Foord et al. (12) and Cameron and Gohar (13). Although the design has improved over the years, the basic principle has remained unaltered and the technique is still widely used nowadays; for example see Cann et al. (2) and Kaneta et al. (14). A typical measurement result is a fringe map, monochromatic or in colour, from which the film thickness can be inferred. However, for an understanding of the operation of a grease-lubricated EHD contact the film thickness in itself is not enough. For example, fluctuations of the film thickness, that is local fluctuations of the surface elastic deformations, as reported above must be associated with fiuctuations in the pressure distribution. These pressure fluctuations, local and/or transient, will influence associated subsurface stresses and thereby the service life of the contact. Furthermore, the pressure fluctuations can induce vibrations in the bearing which will influence the noise level of the bearing, unwanted in, for example, gyro applications, and may have an additional adverse effect on its service life (bearing life). Thus, although film thickness is an essential parameter for lubricated contacts the pressure distribution is also of great interest.

In this investigation the aim is to estimate the pressure distribution in a grease-lubricated contact and to investigate the magnitude of the fluctuations mentioned above. A combined experimental and theoretical approach is introduced in which measured film thickness maps are used as input for pressure computations based on the elastic deformation equation. Wong et al. (15) used a similar approach in their improved version of the impact microviscometer introduced by Paul and Cameron (16). In the impact microviscometer by Wong et al. a steel ball impacts a glass hemisphere and the oil trapped between the ball and the glass deform the surfaces elastically, producing a dimple in the middle of the contact. The shape of the dimple was evaluated using optical interferometry and the pressure distribution in the dimple was calculated using the relation between pressure and elastic deformation. Wong et al. only investigated oil and because of the axial symmetry of their pure squeeze problem, only pressures along the contact radius was estimated, reducing the number of discrete pressures to calculate to about 20 . In the present investigation no symmetry exists and thus the entire contact area has to be mapped using about 4000 pressure points, which puts higher demands on the numerical algorithms.

Evaluating the pressure distribution in a greaselubricated contact using the relation between pressure and elastic deformation has the advantage that the pressure profile in the contact is obtained without having to make any assumption regarding rheological behaviour of the grease. The approach is tested out and verified using experimental and theoretical results for an oillubricated contact. Subsequently it is applied for the purpose stated above, that is to investigate the pressure fluctuations in a grease-lubricated contact.

\section{OPTICAL INTERFEROMETRY MEASUREMENTS}

The ball and disc apparatus used is shown in Fig. 1. The main parts are a steel ball with a diameter of 50 $\mathrm{mm}$ and a glass disc. The disc has a diameter of $100 \mathrm{~mm}$ and a thickness of $6 \mathrm{~mm}$. It is coated with a layer about $200 \AA$ of chromium on the side facing the ball. The ball

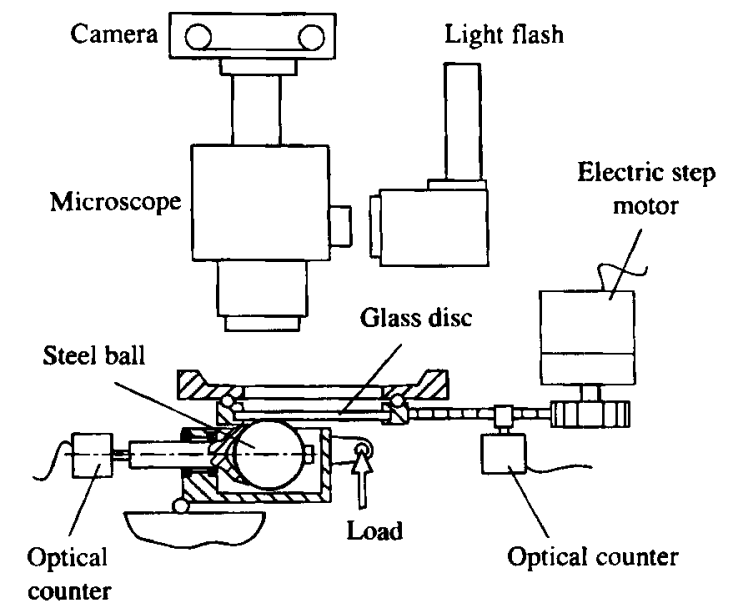

Fig. 1 Ball and disc apparatus 
Table 1 Properties of the lubricants. The grease, G, is thickened with 23 per cent by weight of lithium12-hydroxysterate, giving it the consistency of NLGI grade 2

\begin{tabular}{|c|c|c|c|c|c|}
\hline & $\begin{array}{c}\text { Viscosity at } 20^{\circ} \mathrm{C} \\
\text { and } 150 \mathrm{~s}^{-1}\end{array}$ & $\begin{array}{l}\text { Viscosity at } 20^{\circ} \mathrm{C} \\
\text { and } 4860 \mathrm{~s}^{-1}\end{array}$ & $\begin{array}{c}\begin{array}{c}\text { Pressure viscosity } \\
\text { coefficient }\end{array} \\
\end{array}$ & $\begin{array}{l}\text { Density } \\
\text { at } 15^{\circ} \mathrm{C} \\
\end{array}$ & $\begin{array}{c}\text { Refractive index at } \\
20^{\circ} \mathrm{C} \text { and atmospheric }\end{array}$ \\
\hline Symbol & Pa s & Pa s & $\mathbf{G P a}^{-1}$ & & pressure \\
\hline $\begin{array}{l}O \\
G\end{array}$ & $\begin{array}{c}0.72 \\
15.6\end{array}$ & $\begin{array}{l}0.72 \\
1.46\end{array}$ & $\begin{array}{l}32 \\
17^{*}\end{array}$ & $\begin{array}{l}913 \\
847^{*}\end{array}$ & $\begin{array}{l}0.497 \\
0.467\end{array}$ \\
\hline
\end{tabular}

* For the base oil of the grease.

was rolling on the disc, driven via a belt by an electric step motor, at a rolling speed of $58 \mathrm{~mm} / \mathrm{s}$. The loading was achieved through a pneumatic cylinder, forcing the ball in contact with the disc. The load on the ball was $82 \mathrm{~N}$, resulting in a maximum Hertzian pressure of 430 $\mathrm{MPa}$.

The contact between the ball and the disc was photographed through a microscope and the contact was illuminated with a light flash discharged through the microscope. The flash time was about $0.3 \mathrm{~ms}$, implying that the surfaces moved 3 per cent of the contact diameter during the exposure.

When lubricating the contact with grease, both disc and ball were lubricated before the test and the pictures were taken during the first revolution of the disc. The pictures thus represent full film lubrication. After the first revolution of the disc the contact is gradually starved if grease is not continuously applied to the inlet. The measurements were carried out at room temperature, $23^{\circ} \mathrm{C}$.

Two lubricants were investigated in the ball and disc apparatus, a mineral oil of naphtenic type and a lithium grease with a synthetic poly- $\alpha$-olefin base oil. In the following sections, results related to the oil experiment and calculations will be referred to using ' $O$ '. Alternative ' $G$ ' will be used to refer to results for the greaselubricated contact. Specific details of both lubricants are listed in Table 1.

\section{IMAGE PROCESSING}

The ball-on-disc experiment results in an interferometric fringe picture of the contact. The next step is to obtain from this picture a map of film thickness values, preferably with a small spacing, that is as small as the fringe pattern allows. This conversion process from the fringe pattern to the film thickness map should preferably be done automatically, that is using a computer program including picture processing algorithms.

For the present study the pictures obtained from the ball and disc apparatus were transformed to a photo CD (compact disk) and from that to the computer program. The reason for using a photo $C D$ is that it captures the picture with higher resolution than, for example, a video recording. From the photo $C D$ a picture covering the contact area and its vicinity was taken and about 500 times 500 points from that picture were captured and evaluated in the computer.

A picture processing program [see Gustafsson et al. (17)] calculated the spectral wavelength in each point of the picture, using the local colour information. Subsequently the film thickness map was obtained using the following formulae:

$$
h=\frac{\lambda}{2 n}\left(q+\frac{\Delta \phi}{2 \pi}\right)
$$

In this formula $\lambda$ denotes the wavelength of the light, $\Delta \phi$ the difference between the phase shifts due to reflection on the chromium layer and on the steel ball surface, and $q$ denotes the fringe order. In the equipment used $\Delta \phi$ was calibrated to -1.2 radians, [see also Foord et al. (12)]. The variation in refractive index due to pressure was calculated using the LorenzLorentz relation $(5,12)$.

Each step in the transformation of the interference picture from the original one seen in the microscope to the representation of it in the computer has influence on the colour information. The estimated spectral wavelength calculated in the picture processing program has to be verified with a calibration procedure performed under the same conditions as the actual measurement. In the present investigation the verification of the spectral wavelength was performed in the same way as the calibration of the phase shift difference. In a film thickness interval between 0.1 and $0.7 \mu \mathrm{m}$, the accuracy of the estimated spectral wavelength was very good. The error in the film thickness evaluation stemming from the image analysis is believed to be small compared to other sources of error like the estimation of the refractive index.

The main problem in the entire conversion procedure as described here was the assignment of the fringe order. For the results presented below this assignment was done by hand. As a consequence of this manual step, the film thickness map was limited to 65 times 65 points which were subsequently used in the pressure calculations as described below. The resulting resolution, however, is nevertheless expected to be sufficient for the present study, as the film thickness disturbances observed in grease-lubricated contacts are rather large and therefore relatively smooth pressure fluctuations are anticipated.

\section{Pressure calculation}

Numerical simulation nowdays is a well-established tool to investigate oil-lubricated EHD contacts [see, for example, references (18) to (21)]. However, numerical simulations of grease-lubricated contacts are rare, and the few models and algorithms that have been presented are of questionable applicability for the present study, as they rely on the Reynolds equation and characterization of the grease behaviour in a global way, that is as if it could be regarded as a fluid (7-11). These assumptions are not likely to hold in the case of soap formations entering the contact. This leaves only two alternative ways to obtain information about the pressure fluctuations: direct measurement of the pressure or of the use of measured film thickness results. This latter approach was adopted here.

Regardless of lubricant flow or rheological behaviour, 
the film thickness in an EHD contact will be the sum of the undeformed gap geometry and the elastic deformation of the surfaces. Hence, it can be modelled as

$$
\begin{aligned}
H(X, Y)= & H_{00}+G(X, Y)+\frac{2}{\pi^{2}} \\
& \times \int_{-\infty}^{\infty} \int_{-\infty}^{\infty} \frac{P\left(X^{\prime}, Y^{\prime}\right) \mathrm{d} X^{\prime} \mathrm{d} Y^{\prime}}{\sqrt{\left\{\left(X-X^{\prime}\right)^{2}+\left(Y-Y^{\prime}\right)^{2}\right\}}}
\end{aligned}
$$

Furthermore, force balance is likely to be satisfied:

$$
\int_{-\infty}^{\infty} \int_{-\infty}^{\infty} P(X, Y) \mathrm{d} X \mathrm{~d} Y-\frac{2 \pi}{3}=0
$$

From these two equations, once the film thickness is given, the pressure can be solved. In fact, as these equations are exactly the ones used to describe the so-called dry contact, this problem is not new. The only difference is that in the dry contact the gap is closed $(H=0)$ in the region of positive pressures whereas here the gap has a specific non-zero geometry. As a result, with some minor modifications [see reference (22)] the multi-level algorithm as presented by Lubrecht and Ioannides (23) to solve the dry contact problem can be used here.

Summarizing, the problem to be solved can be stated as follows. Given a film thickness $H(X, Y)$ and an undeformed geometry $G(X, Y)$ find the pressure distribution $P(X, Y)$ that satisfies equation (2), subject to the boundary conditions $P=0$ and to the condition that $P \geq 0$ in the domain ('cavitation'). The constant $H_{00}$ in equation (2) is determined by force balance.

For the numerical solution equations (2) and (3) were discretized, assuming piecewise constant pressures; for example see Lubrecht and Ioannides (23) and Venner and ten Napel (20). The discretization error in this case is of order $d^{2}$ if $d$ is the grid spacing.

\section{RESULTS AND DISCUSSION}

With the ball and disc apparatus and the image analysis program, film thickness maps were evaluated for one oil, $\mathrm{O}$, and one grease, $\mathrm{G}$, both described in Table 1 . The film thickness map from the grease-lubricated contact contains disturbances caused by soap-thickener formations. Using the film thickness maps, consisting of 65 times 65 points, pressure maps were calculated for both the oil- and the grease-lubricated contact.

\subsection{Oil-lubricated contact}

Given the right input, such as speed, load and viscosity, the film thickness and the pressure in the oil-lubricated contact can be solved numerically. In the present study the solver developed by Venner $(20,21)$ was used to verify the measured film thickness and the pressure calculated from the film thickness. Contour plots of the results from the numerical solution of film thickness and pressure are presented in Fig. 2.

Figure 3 shows the measured fringe pattern. Figure 4 shows the contour plot obtained from the image processing applied to this fringe pattern and the corresponding pressure calculated as described in Section 4. According to the measurements, the film thickness in
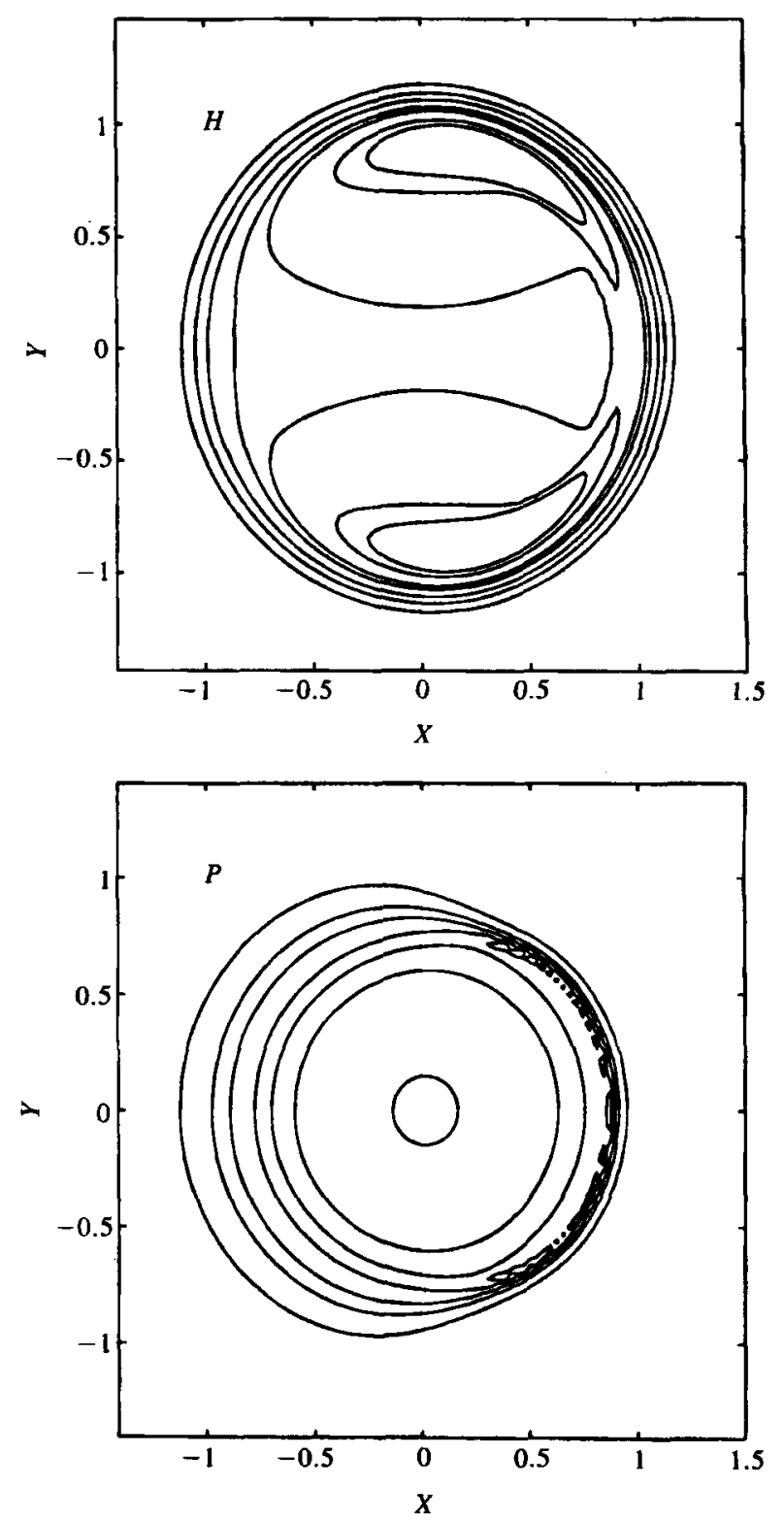

Fig. 2 Film thickness $(H)$ and pressure $(P)$ for the oillubricated contact, calculated using the numerical solver. The solutions were obtained in 513 times 513 points but here only 129 times 129 points are used for presenting the results

the central part of the contact was $0.44 \mu \mathrm{m}$ and the minimum film thickness was $0.26 \mu \mathrm{m}$. This is to be compared with the predictions by the numerical solver which states a central film thickness also of $0.44 \mu \mathrm{m}$ and a minimum film thickness of $0.24 \mu \mathrm{m}$. A comparison between the results from the semi-experimental and the fully numerical procedure is seen in Fig. 5.

As can be seen from Figs 2, 4 and 5, the film thickness from the numerical solutions shows good agreement with the measured film thickness. Acceptable correspondence is also seen between the pressure calculated from the measured film thickness, and the pressure from the numerical solution.

In the contact between the steel ball and glass disc, surface texture will always be present and, even if the steel ball is polished to an $\mathrm{Ra}$ of $0.02 \mu \mathrm{m}$, the surface texture will appear in the film thickness measurements and, consequently, also in the calculated pressure 


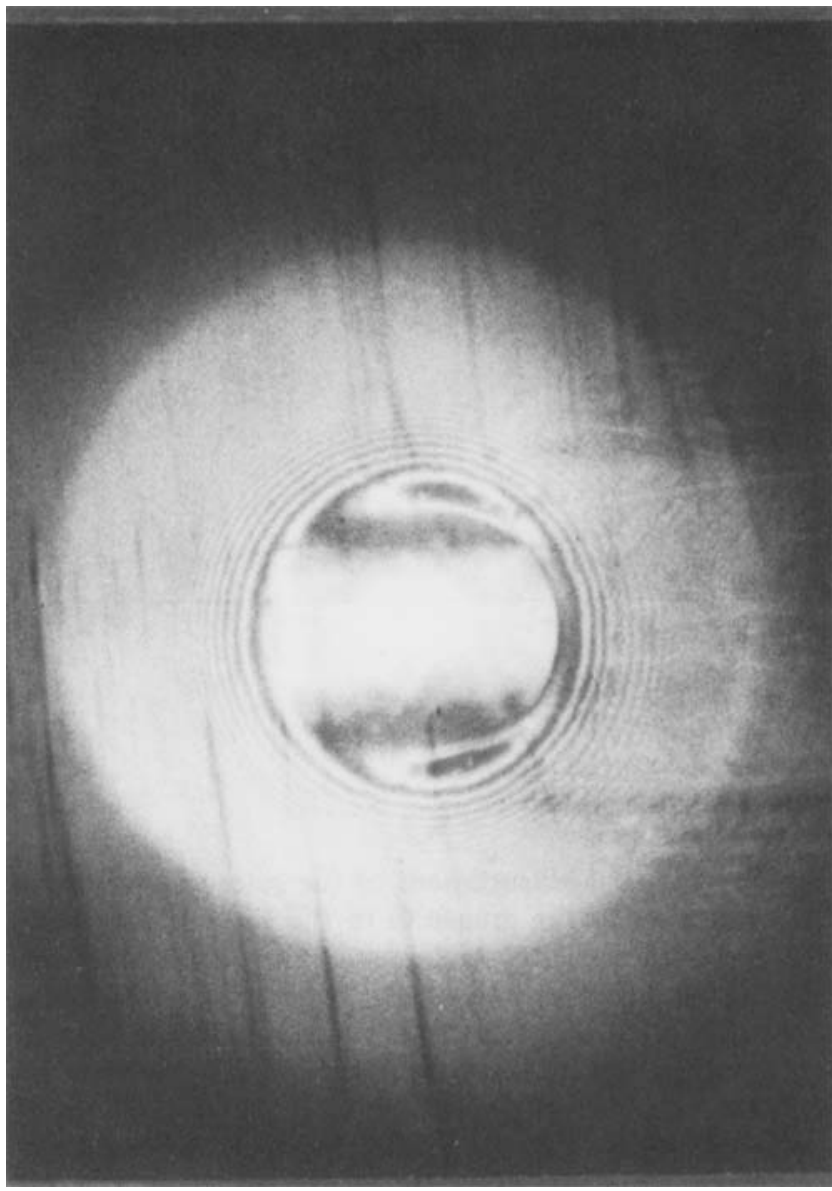

Fig. 3 Reproduction of the original colour slide from the ball and disc apparatus, lubricated with the oil $O$

profile. Some of the small fluctuations in the pressure seen in Fig. 5 are due to the surface texture of the steel ball.

Other effects seen in Fig. 5 are pressure peaks at the contact edges, for example the one at the inlet, $X=-1$. They are due to the discontinuity created in the film

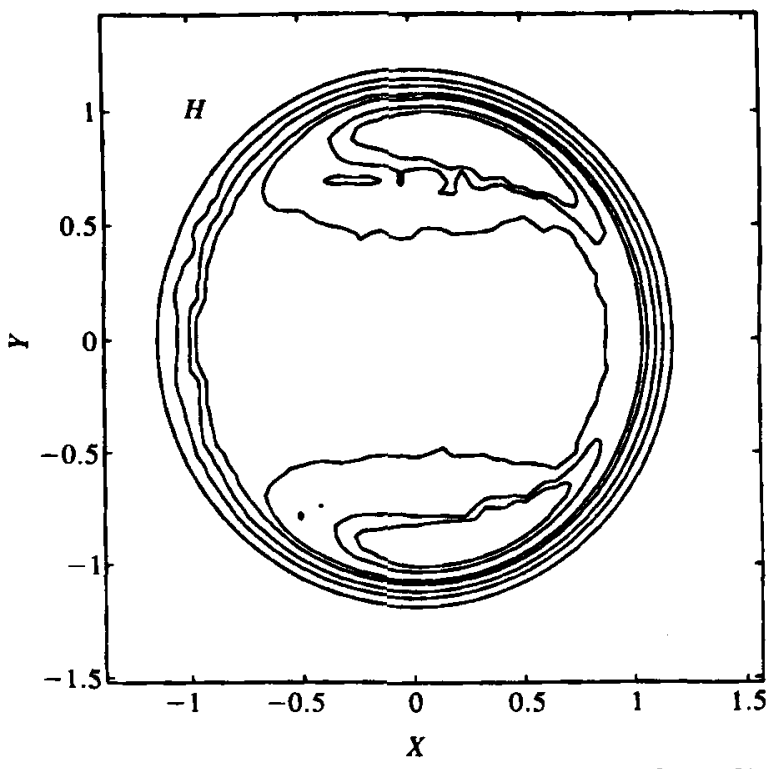

thickness map when supplementing the values obtained from the fringe pattern with values from the geometric surface separation. This transition is needed because outside the contact the surface separation will soon increase above the detectable film thickness using white light optical interferometry. As pressure variations due to this error will always be clearly recognizable they need be of no concern.

These kinds of discontinuities in film thickness at the contact edge are observed more easily at the inlet of the contact because pressure is built up in front of the contact edge and cause elastic deformations that make the surface separation diverge from the parabolic approximation.

\subsection{Grease-lubricated contact}

In the previous section it was shown that the result predicted using the numerical solver correlated well with the measured film thickness map. However, film thickness and pressure profiles of the grease-lubricated contact cannot be obtained numerically. Therefore, reliance is placed on the verification of the model for the oil-lubricated contact, in order to judge which fluctuations in film thickness and pressure are significant. Figure 6 shows the measured fringe pattern for the grease-lubricated contact. Figure 7 shows the contour plot of the film thickness obtained from Fig. 6 using image processing, and the pressure calculated from this film thickness.

The film thickness map in Fig. 7 is distorted compared to the normal horseshoe-shaped film thickness of the oil-lubricated contact (see Figs 2, 3, and 4). Several local changes in film thickness can be observed, like the areas marked $\mathbf{A}$ and $\mathbf{B}$, which show a local increase in the film thickness. This is due to soap-thickener formations in the contact area.

The film thickness disturbances $A$ and $B$ are located at the peripheral part of the contact. In Fig. 8 it can be seen that disturbance $B$ causes a pressure increase of about 25 per cent over the Herzian pressure at that point, reaching the maximum Hertzian pressure found

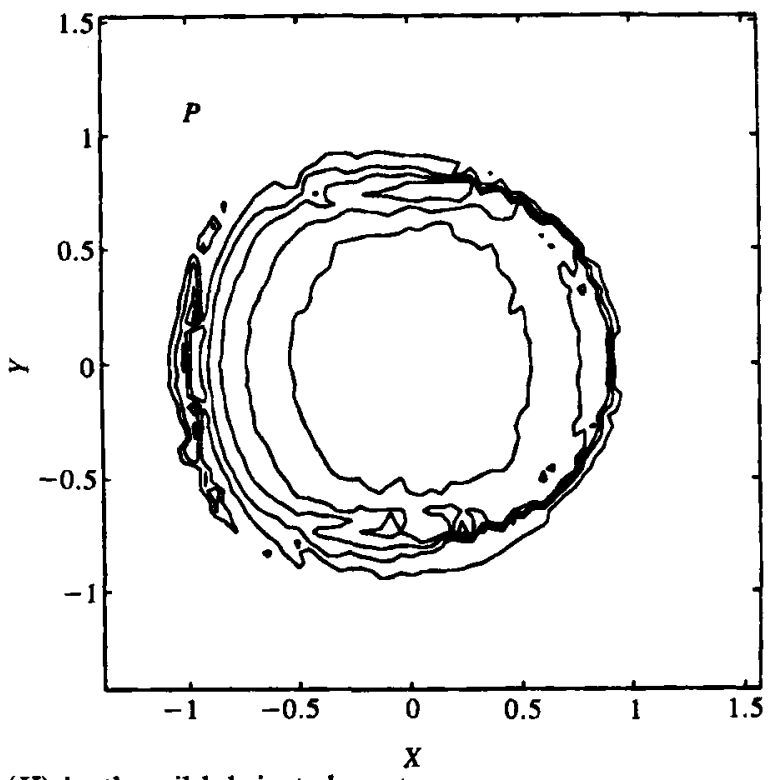

Fig. 4 Contour plot of the film thickness $(H)$ in the oil-lubricated contact, obtained from Fig. 3, and pressure $(P)$ calculated from this film thickness 

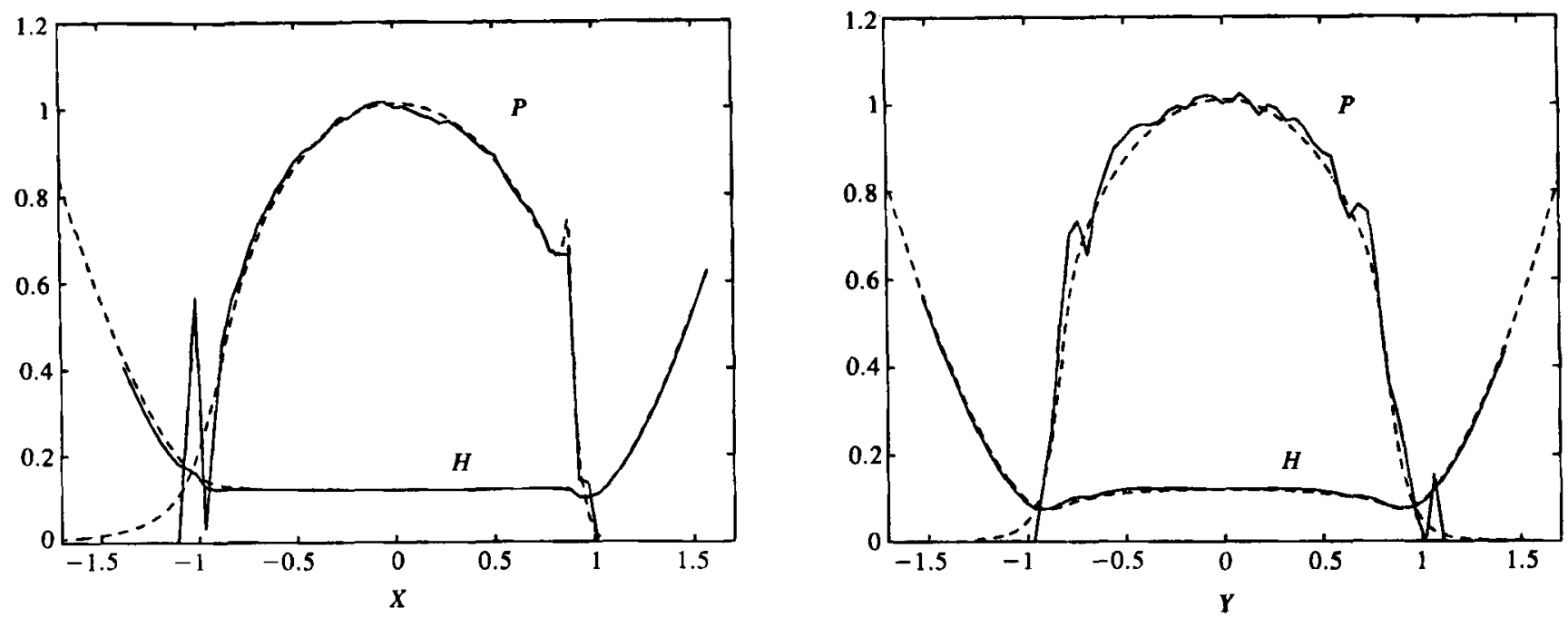

Fig. 5 Film thickness and calculated pressure at $X=0$ (left) and $Y=0$ (right), for the oil-lubricated contact (solid lines). For comparison the results from the full numerical solution are also shown (dashed lines). Pressure and film thickness are presented in dimensionless form

in the contact centre, $0.43 \mathrm{GPa}$. A film disturbance of similar size and magnitude as $B$ but located in the centre of the contact would result in a local pressure increase of 35 per cent or, in this case, up to $0.58 \mathrm{GPa}$.

The thickener formations have higher soap-thickener concentration than the surrounding bulk grease and thus the formations are expected to produce a higher

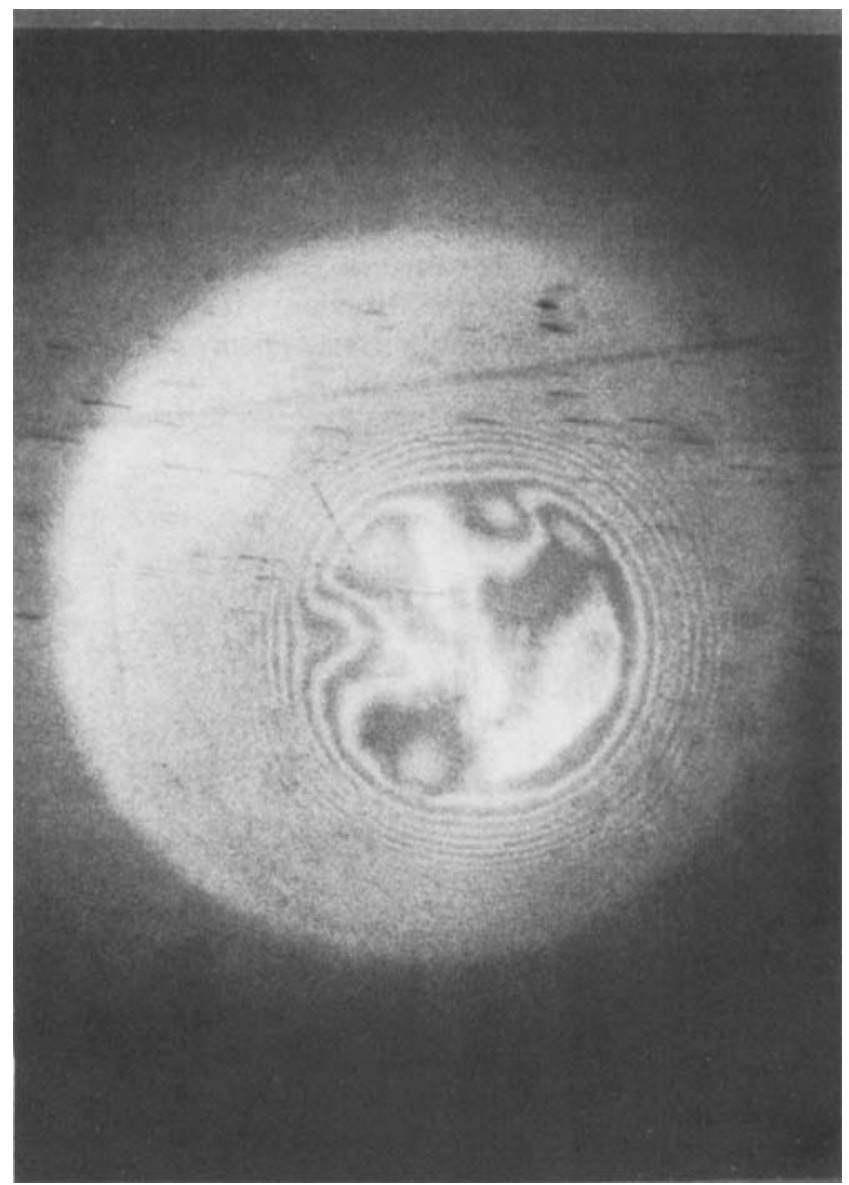

Fig. 6 Reproduction of the original colour slide from the ball and disc apparatus, lubricated with the grease $G$ refractive index. Measurement of the refractive index of greases similar to the grease $G$ in the present investigation, but with varying thickener content [see reference (6)], show, however, that an increase in thickener content from 0 (base oil) to 33 per cent by weight (a very hard grease) make the refractive index increase by less than 1 per cent. A local increase in the refractive index will imply a local overestimation of the evaluated film thickness, in this case by 1 per cent. A conservative estimate of the accuracy of the evaluated film thickness, due to these variations in refractive index, would be \pm 2 per cent.

All variations in film thickness are reflected in the pressure distribution and a significant film thickness fluctuation would mean a significant variation in pressure. The pressure distribution is also sensitive to the film thickness gradient. Abrupt film thickness changes will induce pressure peaks in the pressure distribution, seen, for example, in Fig. 5.

The pressure fluctuations found are significant. With the grease used in this investigation, $G$, the film thickness profile will be dramatically distorted, inducing significant pressure peaks that are about 30 per cent higher than the Hertzian pressure.

Finally, this method for pressure calculation in grease-lubricated EHD contacts gives meaningful results, provided that pressure fluctuations from surface textures and film discontinuities due to the film thickness evaluation are interpreted in the correct way.

\section{CONCLUSIONS}

Due to a lack of an alternative in the form of full numerical simulations, calculating the pressure distribution in a grease-lubricated contact from a measured film thickness profile is a suitable approach. When applied in an investigation of soap-thickener induced pressure fluctuations, as in the present paper, care must be taken in interpreting the results, that is to distinguish them from the fluctuations caused by surface textures and errors in the film thickness map of the contact originating from various sources, for example limited knowledge of used parameter values, etc.

C IMechE 1994 

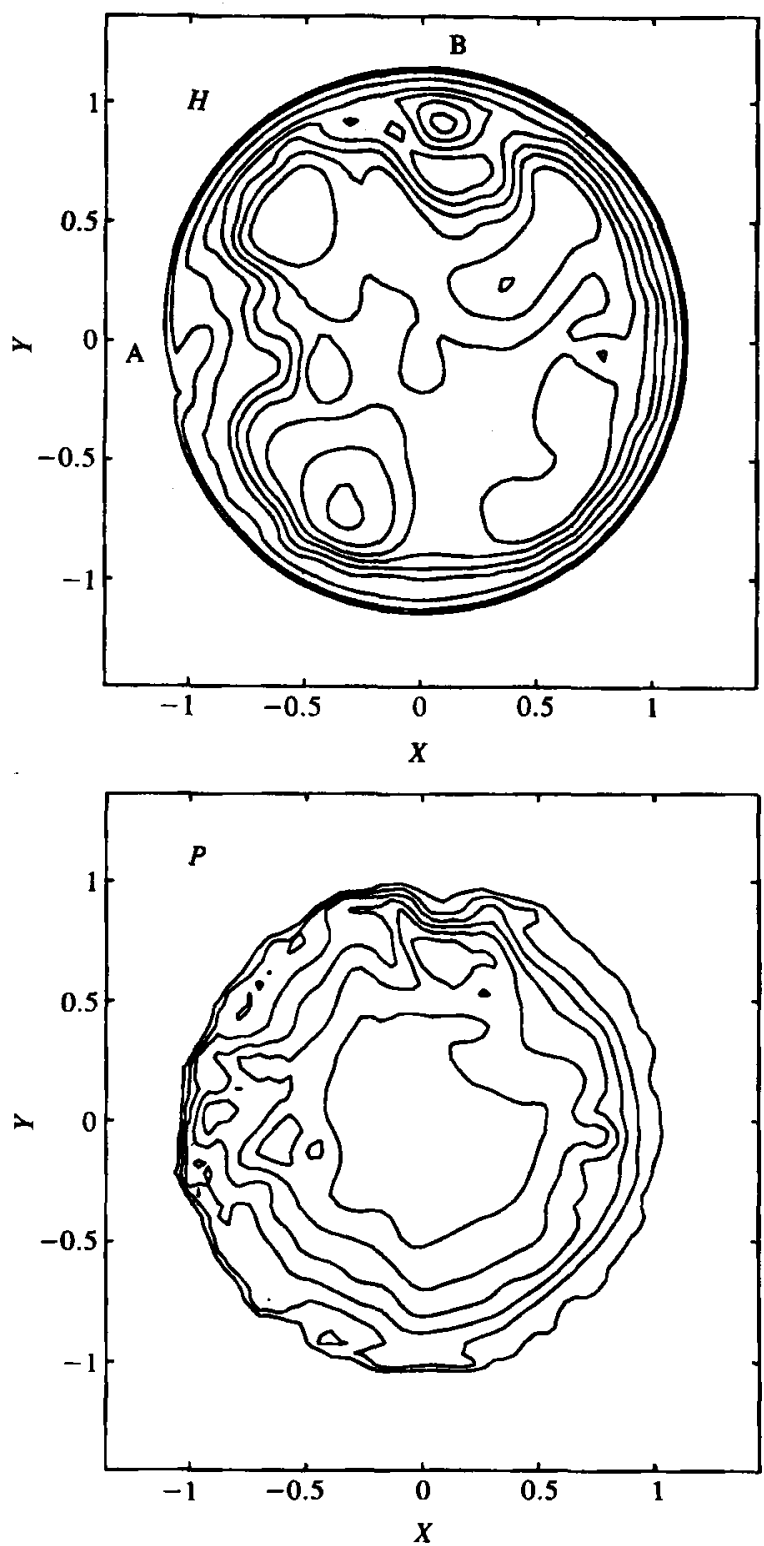

Fig. 7 Contour plot of the film thickness $(H)$ for the greaselubricated contact, obtained from Fig. 6 , and pressure $(P)$ calculated from this film thickness. A and B refer to areas of local film thickness increase, caused by soap-thickener formations

At some points the presented approach needs to be improved, for example automated fringe order assignment in the image analysis phase such that film thickness maps with higher resolution and extending over a larger region can be obtained. This would prevent errors such as the pressure peaks due to matching of measured film thickness and undeformed geometry in the outer region (see Section 5).

With the grease studied here, a lithium grease with a synthetic base oil, local pressure peaks about 25 per cent above the Hertzian pressure were found. If the measured local increase in film thickness appeared in the centre of the contact, instead of at the periphery, with unchanged geometry, the pressure peak was 35 per cent higher than the maximum Hertzian pressure.

\section{ACKNOWLEDGEMENTS}

The authors are grateful for their valuable discussions with Professor Erik Höglund and would like to express
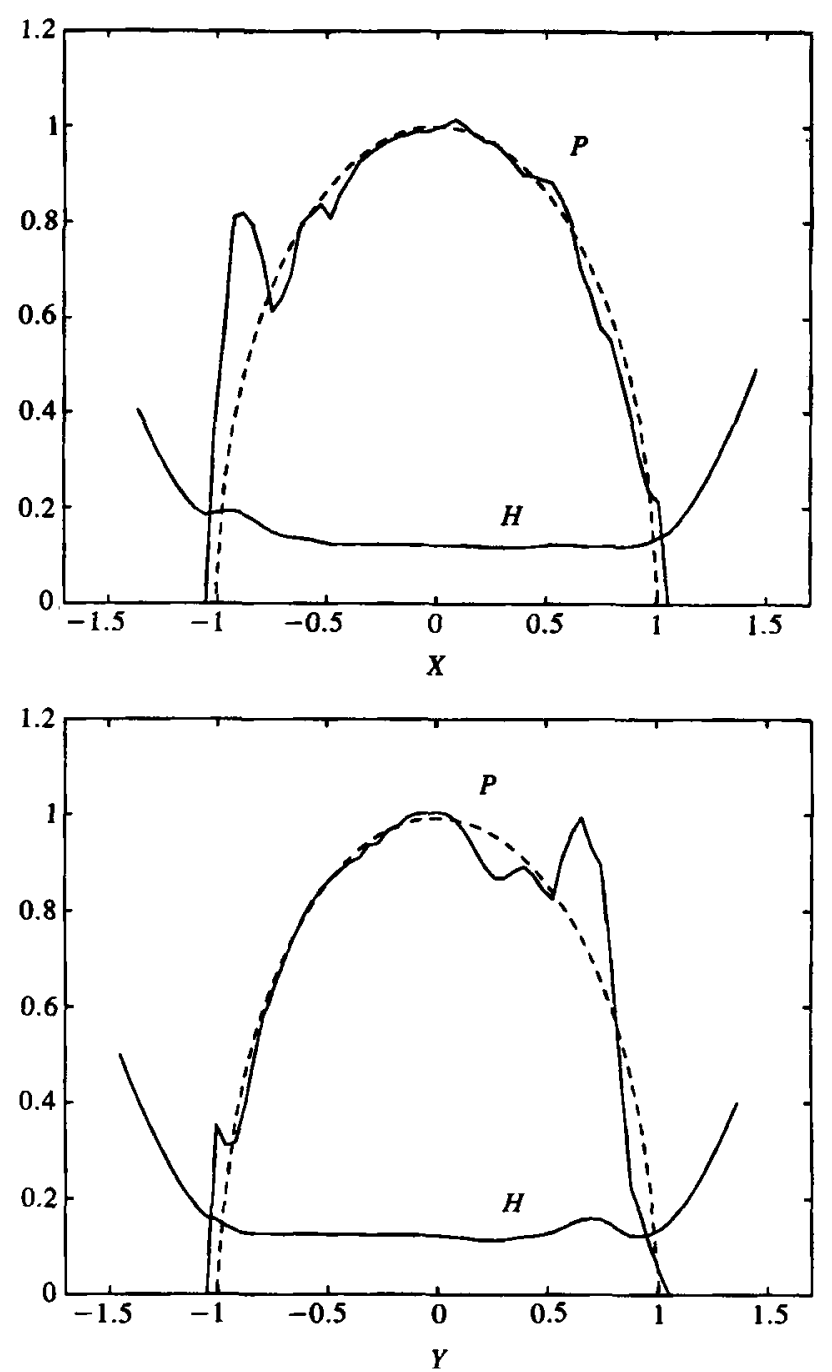

Fig. 8 Film thickness and calculated pressure at $X=0$ (top) and $Y=0$ (bottom), for the grease-lubricated contact (solid lines). For comparison the dry Hertzian pressure distribution is also shown (dashed line). Pressure and film thickness are presented in dimensionless form

their gratitude to Mr Olov Marklund and Dr Lennart Gustafsson for help with the image analysis. The authors also wish to acknowledge the financial support of the Swedish National Board for Industrial and Technical Development (NUTEK) and of the Royal Netherlands Academy of Arts and Sciences, as well as the assistance of Axel Christiernsson Company Limited in preparing the grease.

\section{REFERENCES}

1 Kageymama, H., Machidori, W. and Moriuchi, T. Grease lubrication in elastohydrodynamic contacts. NGLI Spokesman, June 1984, pp. $72-81$.

2 Cann, P. M., Aderin, M. and Spikes, H. A. Optical and infrared studies of the behaviour of grease in lubricated contacts. ELGI Eurogrease, August 1991.

3 Cann, P. M. and Spikes, H. A. In lubro studies of lubricants in EHD contacts using FTIR absorption spectroscopy. STLE Tribol. Trans., 1991, 34(2), 248-256.

4 Cann, P. M. and Spikes, H. A. Fourier-transform infrared study of the behaviour of grease in lubricated contacts. Presented at the Forty-sixth Annual Meeting, Montreal, Quebec, Canada, 29 April -2 May 1991, STLE preprints 91-AM-1B-1.

Proc Instn Mech Engrs Vol 208 
5 Asström, H., Isaksson, O. and Höglund, E. Video recordings of an EHD point contact lubricated with grease. Tribol. Int., 1991, 91(3), 179-184.

6 Aström, H. Irregular film thickness fluctuations in elastohydrodynamic lubrication of a point contact lubricated with grease. Tribologia, 1992, 11(4), 115-122.

7 Dalmaz, G. and Chaomleffel, J. P. Grease film thickness and traction in elastorheodynamic point contacts. ELGI Eurogrease, January 1993, pp. 41-55.

8 Bordenet, L., Vergne, F., Chaomleffel, J. P. and Dalmaz, G. A study of grease film thicknesses in elastohydrodynamic rolling point contacts. Lubric. Sci. 1990, 2(4), 273-284.

9 Dong, D. and Qian, X. A theory of elastohydrodynamic greaselubricated line contact based on a refined rheological model. Tribol. Int., 1988, 21(5), 261-267.

10 Jonkisz, W. and Krzeminski-Freda, $\mathbf{H}$. The properties of elastohydrodynamic grease films. Wear, 1982, 77, 277-285.

11 Jonkisz, W. and Krzeminski-Freda, H. Pressure distribution and film shape of an elastohydrodynamic grease film. Wear, 1979, 55, 81-89.

12 Foord, C. A, Wedeven, L. D., Westlake, F. J. and Cameron, A. Optical elastohydrodynamics. Proc. Instn Mech. Engrs, 1969-70, 184(1, No. 28), 487-505.

13 Cameron, A. and Gohar, R. Theoretical and experimental studies of the film in lubricated point contact. Proc. R. Soc. Lond., 1966, Ser. A, 520-536.

14 Kaneta, M., Sakai, T. and Nishikawa, H. Optical interferometric observations of the effects of a bump on point contact EHL. Trans. ASME, J. Tribol., 1992, 114, 779-784.
15 Wong, P. L., Lingard, S. and Cameron, A. The high pressure impact microviscometer. STLE Tribol. Trans., 1992, 35, 500-508.

16 Paul, G. R. and Cameron, A. An absolute high-pressure microviscometer based on refractive index. Proc. R. Soc. Lond., 1972, A331, 171.

17 Gustafsson, L, Höglund, E. and Marklund, O. Measuring lubricant film thickness with image analysis. Proc. Instn Mech. Engrs, Part J, 1994, 208(J3), 199-205.

18 Hamrock, B. J. and Dowson, D. Isothermal elastohydrodynamic lubrication of point contacts, Part 1. Theoretical formulation. Trans. ASME, J. Lubric. Technol., 1976, 98(2), 223-229.

19 Lubrecht, A. A. ten Napel, W. E. and Bosma, R. Multigrid, an alternative method of solution for two-dimensional elastohydrodynamically lubricated point contact calculations. Trans. ASME, J. Tribol., 1987, 109, 437-443.

20 Venner, C. H. and ten Napel, W. E. Multilevel solution of the elastohydrodynamically lubricated circular contact problem. Part 1: theory and numerical algorithm. Wear, 1992, 152, 351-367.

21 Venner, C. H. and ten Napel, W. E. Multilevel solution of the elastohydrodynamically lubricated circular contact problem. Part 2 : smooth surface results. Wear, 1992, 152, 369-381.

22 Aström, H. and Venner, C. H. A multilevel solver for calculation of pressure from measured film thickness in a grease lubricated EHL point contact. Research report, Lulea University of Technology, Luleà, Sweden, 1993.

23 Lubrecht, A. A. and Ioannides, E. A fast solution of the dry contact problem and the associated sub-surface stress field, using multilevel techniques. Trans. ASME, J. Tribol., 1991, 113, 128-132. 